\title{
Brandaris 128: A digital 25 million frames per second camera with 128 highly sensitive frames
}

\author{
Chien Ting Chin, Charles Lancée, Jerome Borsboom, Frits Mastik, Martijn E. Frijlink, \\ and Nico de Jong ${ }^{\text {a) }}$ \\ Department of Experimental Echocardiography, Erasmus Medical Centre, Rotterdam, The Netherlands \\ Michel Versluis and Detlef Lohse \\ Physics of Fluids, University of Twente, Enschede, The Netherlands
}

(Received 27 May 2003; accepted 21 September 2003)

\begin{abstract}
A high-speed camera that combines a customized rotating mirror camera frame with charge coupled device (CCD) image detectors and is practically fully operated by computer control was constructed. High sensitivity CCDs are used so that image intensifiers, which would degrade image quality, are not necessary. Customized electronics and instruments were used to improve the flexibility and control precisely the image acquisition process. A full sequence of 128 consecutive image frames with $500 \times 292$ pixels each can be acquired at a maximum frame rate of 25 million frames/s. Full sequences can be repeated every $20 \mathrm{~ms}$, and six full sequences can be stored on the in-camera memory buffer. A high-speed communication link to a computer allows each full sequence of about 20 Mbytes to be stored on a hard disk in less than $1 \mathrm{~s}$. The sensitivity of the camera has an equivalent International Standards Organization number of 2500. Resolution was measured to be 36 $\mathrm{lp} / \mathrm{mm}$ on the detector plane of the camera, while under a microscope a bar pattern of $400 \mathrm{~nm}$ spacing line pairs could be resolved. Some high-speed events recorded with this camera, dubbed Brandaris 128, are presented. (c) 2003 American Institute of Physics. [DOI: 10.1063/1.1626013]
\end{abstract}

\section{INTRODUCTION}

High-speed imaging allows fast motion or flow phenomena to be visualized on a slow-motion basis. High-speed imaging is used in the automotive industry (collision and airbag inflation studies) and process control (wrapping and folding, conveyor belt systems, and inkjet printing). It is also used intensively in mechanical engineering (crack studies, combustion, cavitation) and ballistics (impact, ignition, and explosion). High-speed imaging is also applied in various ways in scientific research. ${ }^{1-7}$ Of note are applications in the development of emerging biomedical technologies such as drug uptake by cells, ${ }^{8-10}$ kidney stone fragmentation, ${ }^{11,12}$ and ultrasound contrast imaging. ${ }^{13-16}$

High-speed camera systems come in a variety of configurations. A set of parameters such as frame rate, number of frames, resolution or pixel size, and experimental and financial constraints determines the type of system optimally suited for the application. Commercially available digital high-speed cameras are capable of acquiring 1000 frames per second (fps) at a superb $1000 \times 1000$ pixels resolution. Some of these charge coupled device (CCD)-based and complementary metal-oxide-semiconductor-based systems can achieve higher frame rates by rapidly shifting the charge to neighboring cells, either resulting in a considerable reduction in resolution $(32 \times 32$ pixels at $100 \mathrm{kfps})$ or in a reduction in fill ratio $\left(312 \times 260\right.$ pixels at $1 \mathrm{Mfps} ; 13 \%$ fill ratio). ${ }^{17}$

By employing multiple synchronized cameras, framing

\footnotetext{
a) Author to whom correspondence should be addressed; electronic mail: n.dejong@erasmusmc.nl
}

cameras are used to achieve maximum frame rates between 0.1 and 200 Mfps. This principle in fact predates cinematography and was used in 1878 by Muybridge ${ }^{18}$ in the famous first framing recording of a galloping horse, employing 12 separate cameras. Later, Brixner ${ }^{19}$ used a similar technique with 37 cameras to record the first nuclear explosion at a 1 ms interframe time. Typical framing cameras split the incoming image into several optical channels either by beam splitters, dichroic mirrors, prisms, or a pyramid. Four-to-eight channels are typical and a shutter and an image sensor are mounted in each channel. Using special shutters, high frame rates are possible; e.g., image intensifiers, which can be gated down to $5 \mathrm{~ns}$, are used in the Imacon 468 system (DRS Techonogies, Tring, UK) to achieve frame rates of $200 \mathrm{Mfps}$. The number of frames produced by these systems is limited by the number of independent image intensifier shutters. To increase the number of frames in each record, special techniques can be applied to the image intensifier, such as using a fast phosphor (decay time $=200 \mathrm{~ns}$ ) allowing a second readout within $1 \mathrm{~ms}$, or by dividing the image intensifier into several regions which can be gated separately. ${ }^{20}$ Another system, called the Ultranac, ${ }^{21}$ provides 24 frames at $20 \mathrm{Mfps}$ by sweeping the electron beam in the intensifier.

Higher numbers of frames are available in rotating mirror cameras, where a fast rotating mirror prism sweeps the image along a photographic film mounted on an arc. The original concepts were devised in the late 1930's and the first camera dates back to the first hydrogen bomb explosion in 1952. ${ }^{19}$ The high rotation speed of the mirror allows up to 130 frames to be recorded at a maximum frame rate of 25 Mfps. Photographic films offer excellent image quality, how- 


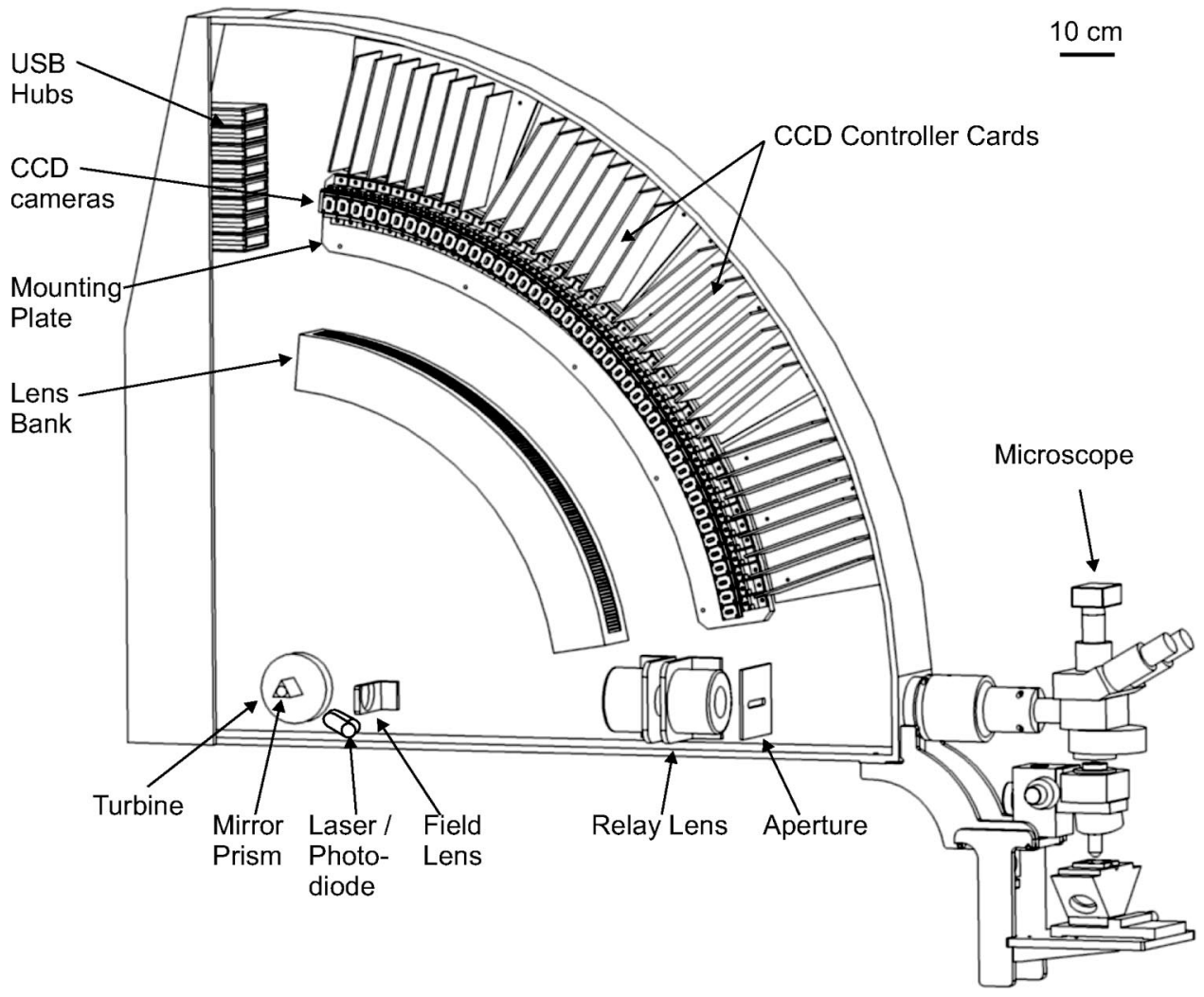

FIG. 1. A drawing of the Brandaris 128 high-speed camera system.

ever, they have some disadvantages. They have limited light sensitivity, reduced flexibility due to film handling and long turnover time due to film developing. In addition, highly specialized shutters, which are both expensive and inconvenient to use, are required at high speeds to prevent multiple exposure of the film.

To address high-speed imaging applications that demand frame rates greater than $1 \mathrm{Mfps}$, at least 100 frames, high sensitivity, high image resolution, operational flexibility, and turnover time of seconds, a digital sensor system based on highly sensitive CCD cameras was developed. In addition, manually operated valves and analog electronics were replaced by computerized controls. This article reports our development of the "Brandaris 128" 22 high-speed framing camera and its characteristics.

\section{SYSTEM DESCRIPTION}

\section{A. Overview}

Brandaris 128, illustrated in Fig. 1, is developed upon the Cordin 119 (Cordin Scientific Imaging, Salt Lake City, Utah) camera frame. The system combines the superior flexibility and sensitivity of electronic CCD detectors with the high frame rate and high number of frames available in rotating mirror cameras. In front of the camera a variety of lenses can be mounted to suit the need of the experiment, for example, Fig. 1 shows a high resolution microscope mounted. Other objectives, such as long-distance microscopes and video camera lenses, have also been used. Generally, a suitable field lens is needed to couple the objective to the Brandaris.

Figure 2 shows a block diagram of the subsystems and associated instruments in a typical experiment. A set of ob- jective and relay lenses projects an image of the target subject onto a mirror prism. A gas turbine spins the three-faced mirror prism at high speed and redirects the incoming light through the lens bank to the CCD detectors. The image is recorded on 128 un-intensified high sensitivity CCD chips mounted on an image arc. Thirty-two CCD Controller Cards $\left(\mathrm{C}^{3}\right)$ control the $128 \mathrm{CCDs}$ and transfer the images to a personal computer (PC) via standard universal serial bus (USB) hubs. The $\mathrm{C}^{3}$ hosts buffer memory that allows six full recordings to be stored on board. An infrared laserphotodiode pair mounted at $30^{\circ}$ below the optical axis generates three mirror pulses per prism rotation. The mirror pulses provide accurate measurement of the instantaneous mirror rotation rate. The mass flow controller (MFC), itself

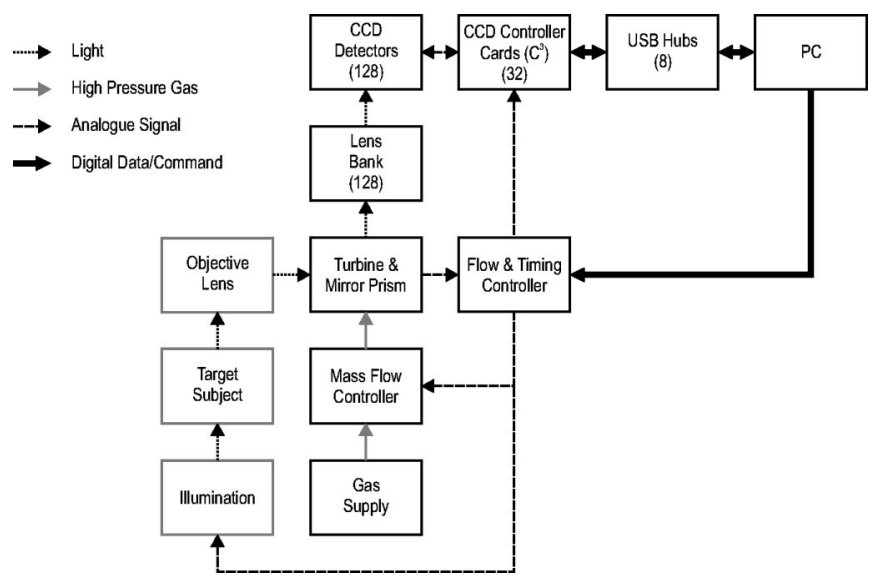

FIG. 2. Block diagram showing the subsystems of the Brandaris 128 in a typical experiment. Blocks outlined in gray represent devices that are external to the Brandaris 128 . 


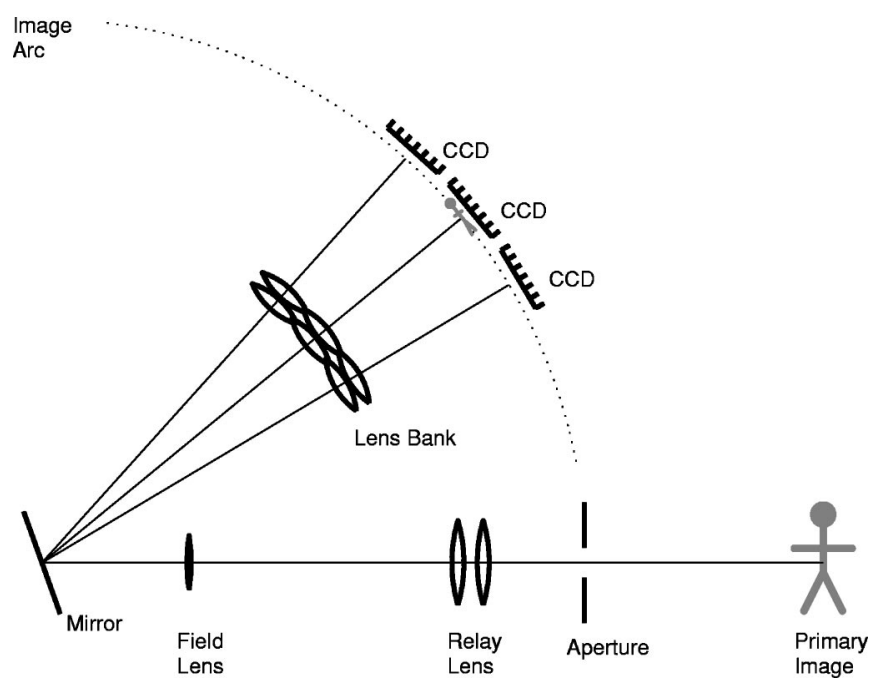

FIG. 3. Optical schematics of a rotating mirror framing camera using the Miller Principle. An object, or a real image, at the primary image plane is imaged by the relay lens onto the rotating mirror. The mirror redirects the beam through sets of lenses in the lens bank which refocus the image onto the CCDs.

controlled by the PC via a flow and timing controller, regulates the gas flow to the turbine and thereby determines the imaging frame rate. The mirror pulses are also used as a master to control the experiment including the timing of the CCDs and the flash illumination source.

\section{B. Optics}

Figure 3 illustrates schematically the optical configuration, which is based on the Miller principle for high-speed cinematography. ${ }^{23}$ The objective lens is mounted on the Brandaris 128 such that a real image is formed on a primary image plane. Alternatively, a real object may be placed directly on the primary image plane. In either case, the dynamic image from the primary image plane is transferred to a sequential series of 128 static images on the image arc by a set of optics.

The relay lenses and field lens form an image of the primary image very near a mirror face of the rotating prism. The mirror face redirects the light beam to successive lens pairs in the lens bank, which refocus the image on CCDs arranged along a $73^{\circ}$ arc. As the rotating mirror sweeps the light beam across successive lenses in the lens bank, the detectors are illuminated for a period of time inversely proportional to the rotational speed of the prism. The aperture limits the extent of the light beam and defines the exposure time as a fixed fraction of the rotational period of the prism. During the illumination of each detector, the image formed on the detector is nearly stationary. The image changes slightly as the mirror rotates (ignoring for the moment any evolution of the image due to the dynamics of the target subject). The resulting blur is minimized by placing the image as close as possible to the apparent axis of rotation of the mirror and by the limiting angular width of each optical channel. The total blur of the final image, called "drag," was calculated following a scheme similar to that given in Igel and Kristiansen. ${ }^{24}$ The result shows that the optical configu-

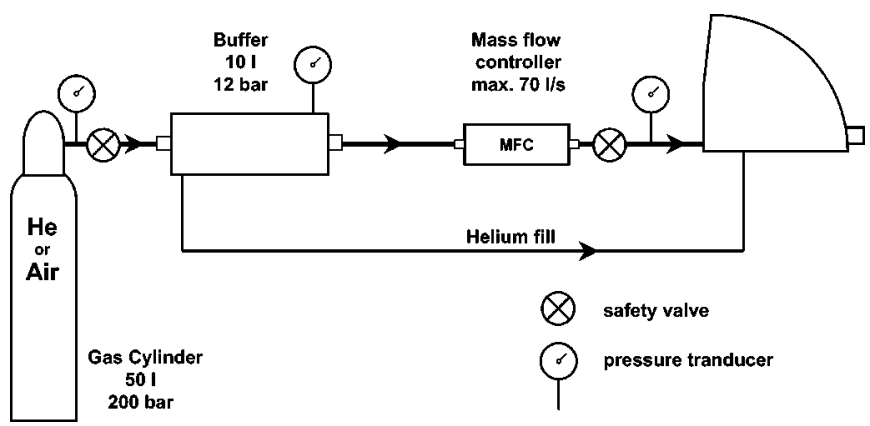

FIG. 4. Schematic diagram of the flow and timing control system. The high pressure helium or air is connected to a buffer at 12 bar. The mass flow controllers regulated the gas at a set flow rate. The mass flow drives the turbine which spins the mirror prism.

ration of the Cordin 119 camera frame produces at the worst case a drag of $7.5 \mu \mathrm{m}$, with $50 \%$ of the channels suffering a drag of less than $4 \mu \mathrm{m}$. These drag values are smaller than both the pixel size of the CCD detectors and the overall optical resolution of the system with a fixed mirror.

The field lens is shifted to either of two preset positions in order to compensate for the change in reflective index while operating in different gases. The total magnification factor from the primary image plane to the final image arc is 0.44 .

\section{Flow control system}

The imaging frame rate is proportional to the turbine rotation speed. A specialized high-speed turbine (Cordin Model 1220) is driven by high-pressure helium or air. Air is used for moderate turbine speeds up to 5000 rotations per second (rps), corresponding to $6 \mathrm{Mfps}$. For higher operational speeds helium is used. The maximum turbine speed is $20000 \mathrm{rps}$, resulting in a maximum frame rate of $25 \mathrm{Mfps}$. The gas flow is controlled by a Bronkhorst (Ruurlo, The Netherlands) F-206AI mass flow controller (MFC), which in turn is controlled by a PC.

The flow system for Brandaris 128 is schematically shown in Fig. 4. The MFC actively regulates the flow rate between 3 and $150 \mathrm{~m}^{3} / \mathrm{h}$. The frame rate and timing parameters are controlled through a program built in the LabView (National Instruments Corp., Austin, TX) environment.

A set of safety valves prevents overpressure and overspeed failure of the turbine/prism system. Finally, a fill line was installed to pre-fill the camera case with helium to reduce viscous drag on the mirror prism during operation at speeds over 5 Mfps.

\section{Detector and CCD controller card}

The central imaging component consists of 128 highsensitivity CCD board cameras (ICX055AL, Sony Corp., Tokyo, Japan). These camera modules measure $27 \mathrm{~mm} \times 40 \mathrm{~mm}$ each with an imaging area of $4.5 \mathrm{~mm} \times 3.6 \mathrm{~mm}$. The distance between adjacent channels on the image arc is $9 \mathrm{~mm}$ and therefore special mounting blocks were designed to mount the 27-mm-wide modules in the limited space. As shown in Fig. 5, one third of the CCDs, channels 2, 5, 8, etc., are placed directly on the image arc. CCDs 1, 4, 7, etc., are 


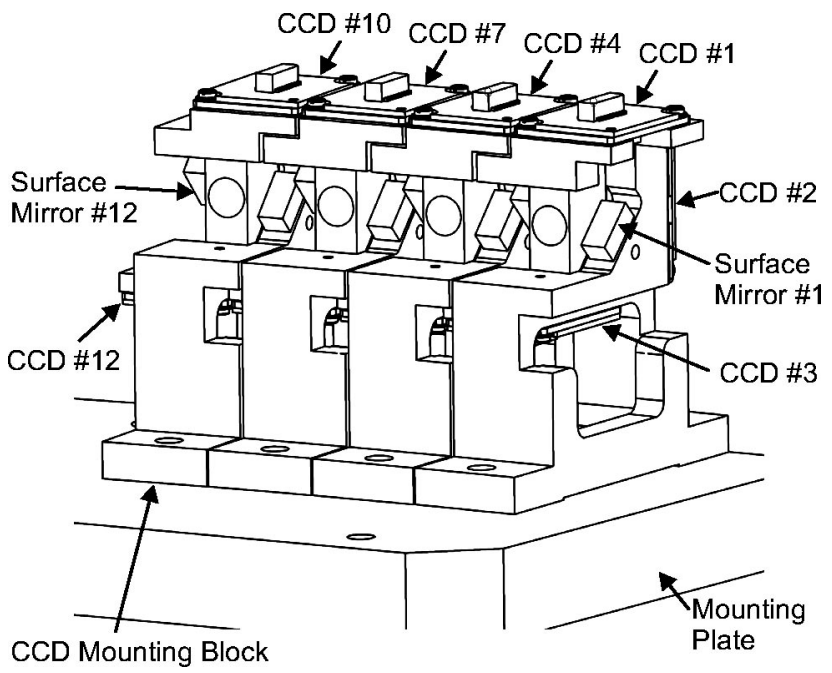

FIG. 5. CCD mounting blocks. In this perspective drawing, the mounting of the detectors for 12 optical channels are shown. Twelve CCDs are mounted on four mounting blocks. CCDs 2, 5, 8, and 11 are mounted directly on the beam path. The rest of the CCDs are mounted on the top or bottom positions. Eight surface mirrors deflect the light beam by $90^{\circ}$ towards these CCDs.

mounted on a top plane perpendicular to the original image plane. CCDs 3, 6, 9, etc., are mounted on a bottom plane also perpendicular to the original image plane. The corresponding images are redirected onto these CCDs with surface mirrors.

The CCD chip has $500 \times 584$ photosensitive cells, each measuring $9 \mu \mathrm{m} \times 6 \mu \mathrm{m}$. The listed dynamic range is approximately 250 times. This sensor has its peak sensitivity at $540 \mathrm{~nm}$ optical wavelength, the $50 \%$ band ranges from 400 to $670 \mathrm{~nm}$. Beyond the visible range, the sensor is sensitive in the near-infrared range up to $1000 \mathrm{~nm}$.

A primary function of the custom designed CCD controller card $\left(\mathrm{C}^{3}\right)$ is to coordinate the capture process of each CCD. A process called flush drains the accumulated photoelectrons in the cells of the CCD down to the grounded substrate. A second process called charge transfer shifts the photoelectrons into the so-called transport channels. There is one transport channel per two photosensitive cells, resulting in $500 \times 292$ effective pixels. The transport channels maintain the photoelectrons until the analogue-to-digital conversion. The digitization of the content of each transport channel is called readout. The beginning and end of exposure are therefore defined by flush and charge transfer, respectively. Flush and charge transfer are performed on all pixels in parallel and require only several microseconds. Readout is performed sequentially on a pixel by pixel basis and requires $20 \mathrm{~ms}$ to finish.

Figure 6 shows the timing diagram of the most standard type of experiment, in which a single event is recorded on a full sequence of 128 frames. When the mirror prism is rotating at a steady rate, the CCD of each channel is illuminated by the mirror at a fixed delay after the mirror pulse generated by the laser-diode pair. To ensure synchronized exposure of the CCDs and prevent multiple exposure, the $\mathrm{C}^{3}$ 's are designed to perform flush and charge transfer when triggered by externally generated timing signals. As seen in Fig. 6, all the CCDs are flushed several microseconds before channel 1

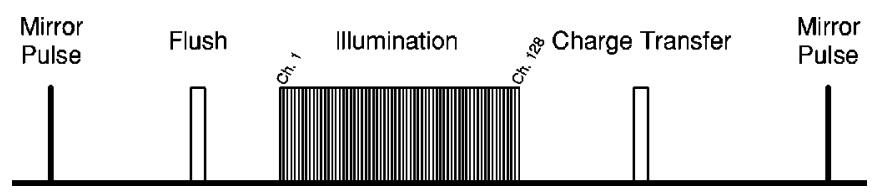

FIG. 6. Timing diagram of an experiment recording a full sequence of 128 images. The CCDs are illuminated by the mirror for about $31 \%$ of the time between successive mirror pulses. The repetition time of the mirror pulses has a range of 17-500 $\mu$ s. In this mode of operation, all CCDs are flushed before the illumination and charge transferred after the illumination. Readout is performed after charge transfer.

is illuminated, and then charge transferred several microseconds after channel 128 is illuminated. Readout is performed after charge transfer.

The $20 \mathrm{~ms}$ duration of readout limits the speed at which recordings can be repeated. To achieve higher repetition rate, a segmented mode was devised. For example, an experiment may be designed to capture 64 consecutive frames in each of two mirror periods. During the first mirror period, the $\mathrm{C}^{3}$ 's flush all the CCDs before illumination and charge transfer channels 1-64 after illumination. During a subsequent, which may be the second, mirror period, the $\mathrm{C}^{3}$ 's again flush all the CCDs before the illumination and then charge transfer channels 65-128 after the illumination. The images frozen in the transport channels of channels 1-64 are not affected by subsequent illuminations and flushes after the charge transfer.

Figure 7 shows the layout of the custom designed $\mathrm{C}^{3}$. Each device is based on an Atmel (San Jose, CA) FPSLIC microcontroller/FPGA chip. The $\mathrm{C}^{3}$ performs the following tasks: (1) flush and charge transfer of 4 CCDs; (2) readout of the CCDs; (3) storing of the data into random access memory (RAM) buffer; and (4) command and data input/ output, in particular transfer the image data, via the USB 2.0 interface. Three hardware signal inputs, start, flush, and transfer, provide synchronization to all the $\mathrm{C}^{3}$ with about 40 ns precision.

After the start signal the microcontroller begins counting transfer pulses until a preset number is reached, at that moment a charge transfer is carried out. Different CCDs can have different preset values. After charge transfer was carried out on all the CCDs, the analog data are read out simultaneously and digitized by four analog-to-digital converters (ADCs). The ADC has a programmable gain of $0-45 \mathrm{~dB}$. The ADC is 10 bits, but only the eight highest bits are used. Furthermore, two different offsets can be programmed; one to correct for the black level on a per-line basis, using the mask cells, the other can be used for automatic shading correction. The on-board RAM buffer allows six images per detector to be stored. At the request from the PC, the data is transferred via the USB 2.0 controller, which has a maximum transfer speed of $480 \mathrm{Mbits} / \mathrm{s}$. However, the maximum measured transfer rate of a single $\mathrm{C}^{3}$ is 5 Mbytes/s, due to the limited speed of the RAM chips. With $32 \mathrm{C}^{3}$ 's working simultaneously, the sustained effective transfer rate was found to be 20 Mbytes/s, allowing a full 128 frame record to be stored on a hard disk within $1 \mathrm{~s}$.

A USB 2.0 device driver of the $\mathrm{C}^{3}$ has been written for the Windows 2000/XP platform. An instruction set that pro- 


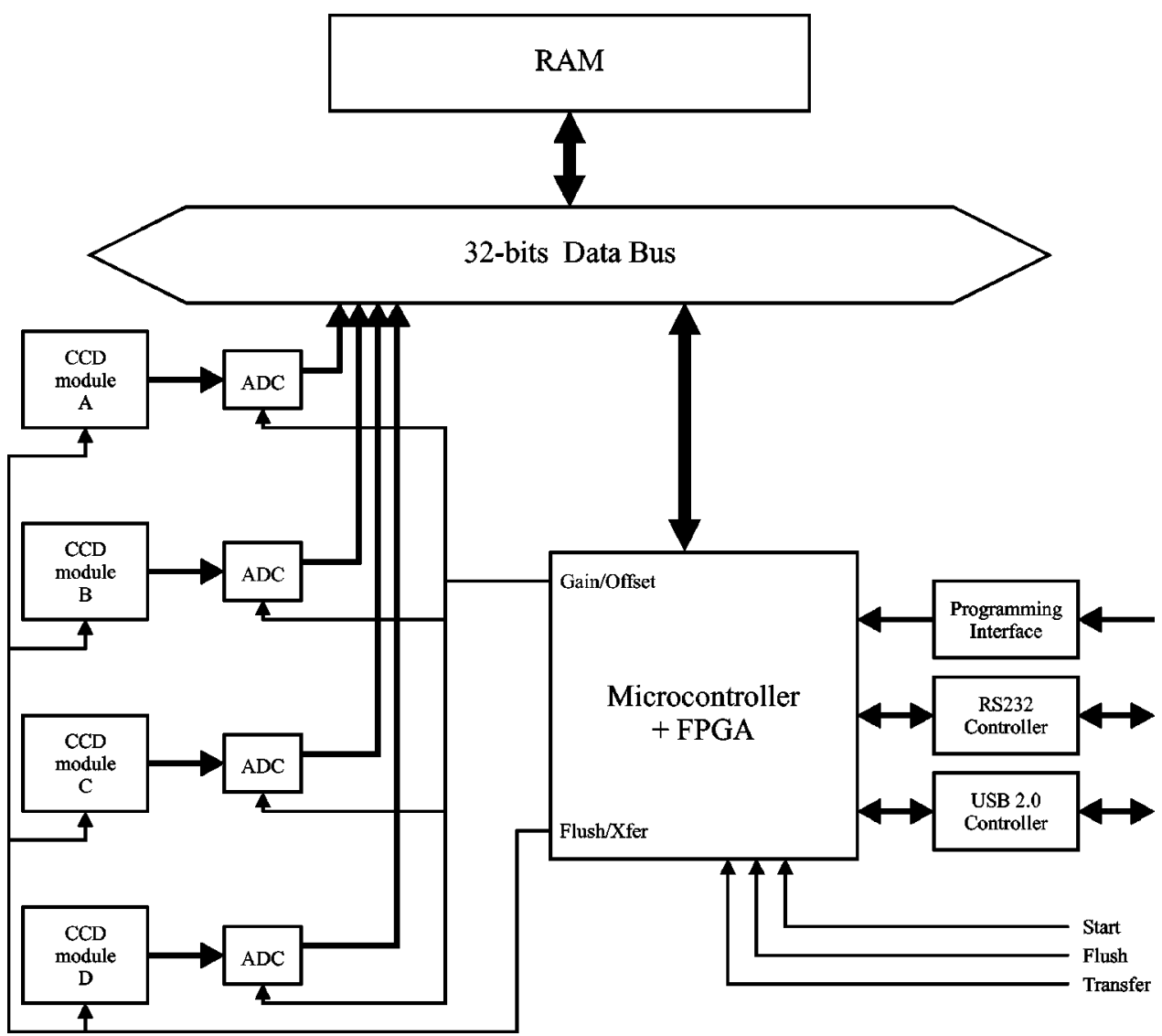

FIG. 7. Block diagram of the CCD controller card $\left(\mathrm{C}^{3}\right)$. Each $\mathrm{C}^{3}$ controls four CCDs. Following software commands transmitted via the USB interface, and in response to hardware trigger signals (Start, Flush, Transfer), actions such as flush, charge transfer and readout are performed at precise timing.

vides complete access to all the functionality of the $\mathrm{C}^{3}$ has been created for the Matlab and $\mathrm{C}$ programming languages. After acquisition, image frames are realigned using affine transformations to correct for small misalignments between the detector channels. ${ }^{25,26}$ The misalignments arise from imperfect positioning of the relay lenses and CCDs, and are static as a result. The correction transformation was predetermined by analyzing a recording of a fixed grid target. The realignment process is independent of the information in the image frames and therefore can be performed quickly. The resulting corrections on the CCD plane were found to be $\pm 38 \mu \mathrm{m}$ (standard deviation) in each direction.

\section{E. Timing controller}

Since the turbine is stable for a given set value of the mass flow, pretriggers can be also derived. The mirror pulse precedes the exposure of the first CCD by $5 \mu$ s at the maxi-

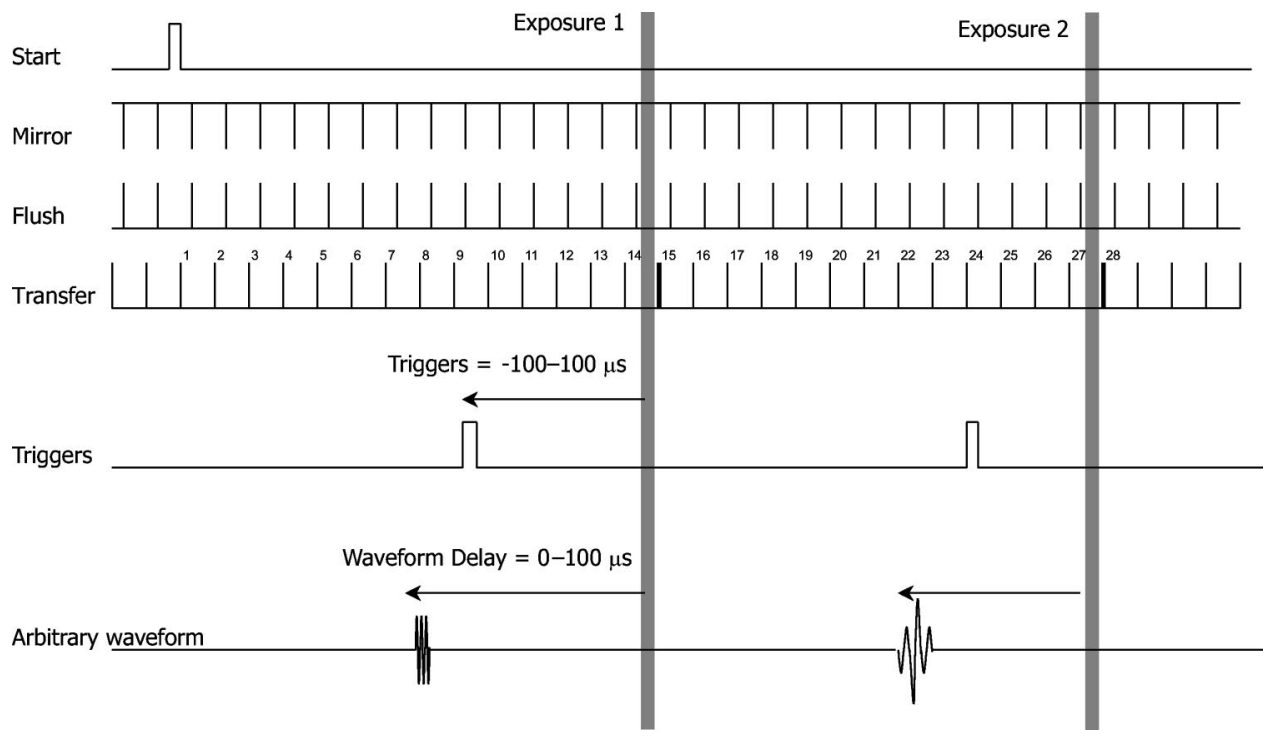

FIG. 8. Timing diagram of a typical experiment. After start signal is detected the transfer pulses are counted. When the count for exposure 1 is reached (15 in this case) the image of the CCDs which has this preprogrammed number is stored. The same process is repeated for exposure 2 when count 28 is reached. A total of up to 48 exposures can be programmed. 


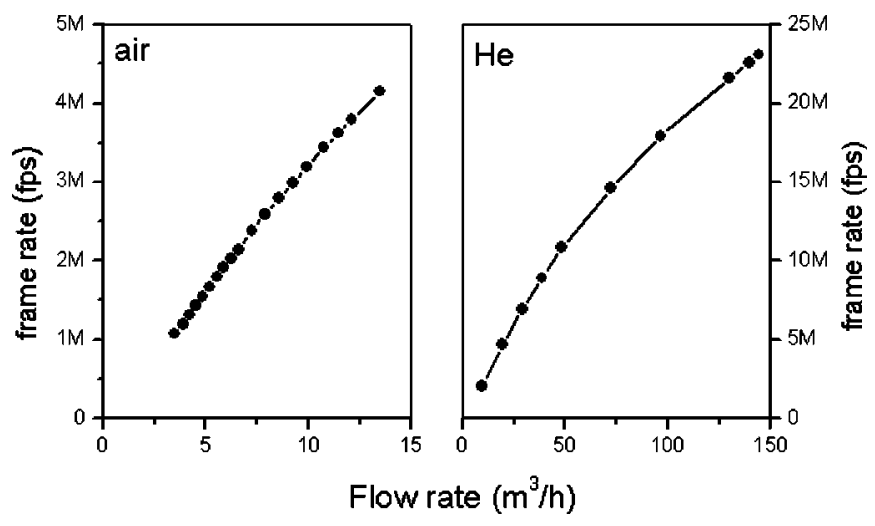

FIG. 9. Frame rate vs volumetric flow rate (at normal condition) of the driving gas using air and helium.

mum frame rate. From the mirror pulses two types of controlling signals are derived as shown in Fig. 8. The first type are the flush and the transfer pulses, which are used by all the $\mathrm{C}^{3}$. Typically the flush pulse precedes the exposure of the first CCD by $10 \mu \mathrm{s}$, the required time to flush the CCDs. The transfer pulse follows after the rotating mirror has illuminated CCD number 128.

The second type of control signals is used to control the experimental equipment external to the camera. These include pre- and post-triggers for flashlight, arbitrary wave form generator, and other devices such as switches and multiplexers.

An experiment consists of 1-48 exposures. For each exposure the number of CCDs to be used and the timing can be independently preprogrammed. For example, six exposures of 128 channels each can be used. In such a case, consecutive exposures have a time gap of $20 \mathrm{~ms}$ minimum, the time required for readout. If consecutive exposures employ different sets of CCDs, then the time gap between exposures can be as short as one mirror period.

Figure 8 shows the timing of a typical experiment. The start signal is the absolute starting time for all the $\mathrm{C}^{3}$ 's. After the start signal is detected the transfer pulses are counted.

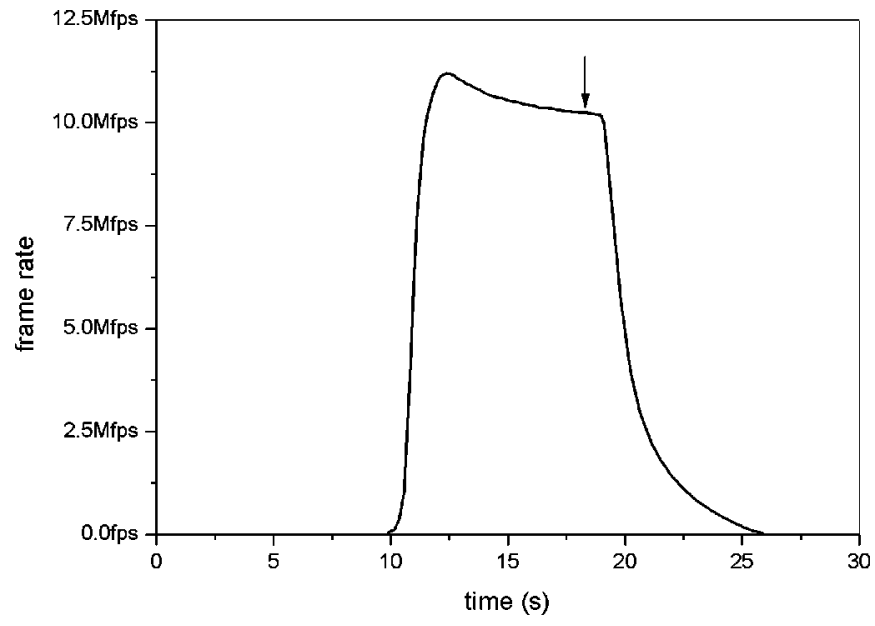

FIG. 10. Turbine response for a typical experiment using helium. The MFC requires $10 \mathrm{~s}$ to initialize, then after a small overshoot, the frame rate was stablized at $10 \mathrm{Mfps}$. At the time indicated by the arrow, six recordings were captured.
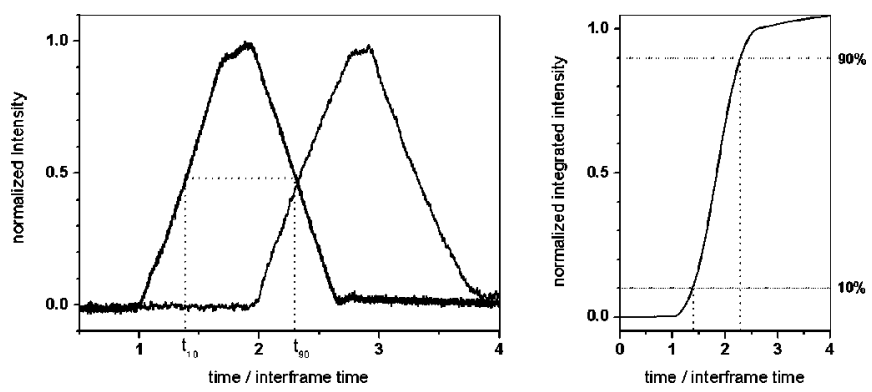

FIG. 11. Measured exposure time of two consecutive channels.

When the count for exposure 1 is reached (15 in this case), the image of the CCDs which has this preprogrammed number is stored. The same process is repeated for subsequent exposures. For each exposure eight pre- and posttriggers as well as two arbitrary wave forms are available to control the experiment.

\section{RESULTS}

\section{A. Flow system}

Figure 9 shows the measured frame rate of the camera as a function of the flow rate of the mass flow controller for air and helium. Figure 10 shows the frame rate as a function of time in a typical experiment. The MFC requires $10-70 \mathrm{~s}$, depending on the chosen frame rate, to initialize after the frame rate is set. The gas flow quickly accelerates the turbine and after a small overshoot the turbine stabilizes to its preset frame rate of $10 \mathrm{Mfps}$, where six recordings of 128 frames can be captured, after which the mass flow is shut off and the turbine decelerates. The overall stability of the system was found to be better than $0.2 \%$ over $5 \mathrm{~min}$ of operation. As parts of the turbine heat up during operation at higher speeds, the operational time is limited to reduce wear and prevent fracture failure of the mirror turbine assembly. At $25 \mathrm{Mfps}$ the camera can be operated for $30 \mathrm{~s}$, below $6 \mathrm{Mfps}$ it can be operated for several minutes.

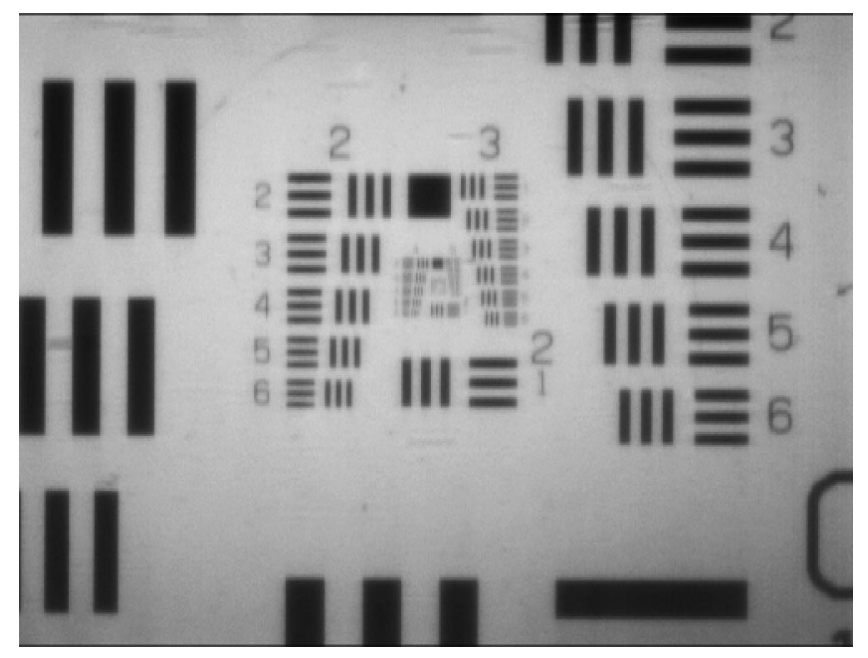

FIG. 12. An image of the USAF 1951 test target for spatial resolution. The resolution was about $36 \mathrm{lp} / \mathrm{mm}$ horizontally and $16 \mathrm{lp} / \mathrm{mm}$ vertically, as measured on the CCD. 

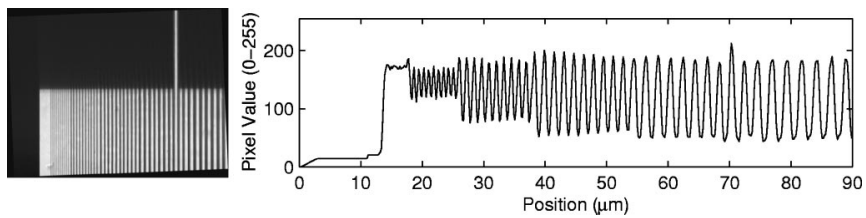

FIG. 13. Image of a test target recorded using a $120 \times$ microscope. Lines with $0.4 \mu \mathrm{m}$ spacing were resolved.

\section{B. Exposure time}

The temporal intensity profiles of two adjacent channels was measured with Siemens (Munich, Germany) SFH-213 fast photodiodes mounted in a CCD mounting block. Figure 11(a) shows the normalized intensities as functions of time in units of the interframe time as independently determined from the actual rotation speed of the mirror. The measurement shows the triangular intensity profile which results from the convolution of two rectangular aperture functions. ${ }^{23,24}$ It also shows that the centers of the intensity profiles are exactly at one interframe time distance. The exposure time of each channel is defined here as $t_{90}-t_{10}$, where $t_{10}$ and $t_{90}$ denote the time points when $10 \%$ and $90 \%$, respectively, of the total light energy is received by the detector. These time points can be inferred from a plot of the integrated intensity as a function of time, see Fig. 11(b). From this analysis it follows that the exposure time is 0.9 times the interframe time. Also seen in Fig. 11(a) is that the intensity level at which $t_{10}$ and $t_{90}$ occur coincides with the crossing of the intensity profiles of the two channels, emphasizing the fact that each individual frame is indeed recorded discretely in time.

\section{Sensitivity}

The optical sensitivity of high frame rate cameras is of great importance since exposure times are extremely short. However, sensitivity for such cameras, if available at all, is often quoted in a form that is difficult to use. Two approaches were used to assess the sensitivity of the Brandaris128. The sensitivity in International Standards Organization (ISO) number of the ICX055AL camera was estimated using a photographic light meter. This measurement provides a comparison to conventional rotating mirror cameras using photographic film. A $50 \mathrm{~mm} \mathrm{f} / 2.8$ projector lens was mounted in front of an individual ICX055AL camera module controlled by a $\mathrm{C}^{3}$. A Melles Griot (Carlsbad, CA) infrared
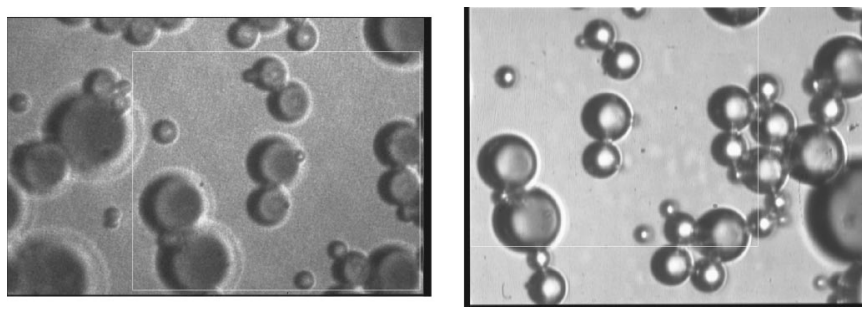

FIG. 14. Comparison of images of the same target captured from an Imacon 468 camera (left) and the Brandaris 128 (right). The magnification was $120 \times$, the field of view (FOV) of the right image was $89 \mu \mathrm{m} \times 68 \mu \mathrm{m}$. Due to difficulty in alignment, the two fields of view are not identical. The white boxes mark the region of the FOV common to both images. filter was mounted in front of the lens to block the infrared part of the spectrum from contributing to the exposure. Diffused room light was used to illuminate an $18 \%$ gray card, and a Profisix (Gossen, Germany) light meter was used to determine the illumination level. The exposure time and ADC gain level on the $\mathrm{C}^{3}$ was controlled interactively using a Matlab program. For each illumination level and gain level, the exposure time was adjusted until an image with average pixel values of half maximum was obtained. From the f-number, the illumination level measured by the light meter and the exposure time needed to create a half-gray image, the ISO number was calculated. ${ }^{27}$ The measured ISO number varies with the gain level, every $6 \mathrm{~dB}$ increase in gain results in a doubling of ISO number. Over the range of available gain levels, the measured ISO number has a range of 400100000 ; at the default gain of $17 \mathrm{~dB}$, the ISO number was 2500.

A second approach to determine the sensitivity was a comparison to an eight channel framing camera, the Imacon 468 (Hadland Photonics, Tring, United Kingdom), which was used in several studies of microbubbles in ultrasound. ${ }^{13-15}$ Using optical density filters, the illumination level was adjusted until each system produced image frames which are detectable. In this case a detectable image is defined as a signal-to-noise-ratio (SNR) level of 2 . In both systems, the exposure time was set at $1.0 \mu$ s and all the available gain levels on each system were tested. The ratio of the illumination intensity resulting in detectable images in each system is then the relative light sensitivity between the two cameras. Using the maximum gain setting on both cameras, our measurement revealed that Brandaris is approximately $50 \%$ as sensitive as the Imacon 468 .

The dynamic range of a camera system is defined as the ratio between the highest light intensity resulting in unsaturated images and the lowest light intensity resulting in detectable images. Under the particular experimental conditions used, the Imacon 468 and Brandaris-128 gave dynamic range levels of 200 and 250, respectively. The dynamic range within an image frame is lower than the full dynamic range, since the gain within a frame is fixed. Imacon 468's image dynamic range is variable due to the nonlinearity of the gain of image intensifiers. A representative value for the Imacon 468 is 16, while a typical Brandaris 128 image frame has a dynamic range of 30 .

\section{Resolution}

To determine the optical resolution of the Brandaris system, a resolution target was placed at the primary image plane. The test target has a glass substrate and a photoetched chrome layer with line patterns. Figure 12 shows the results for the two principal directions of the system. The optical resolution was 36 line pairs per millimeter $(1 \mathrm{p} / \mathrm{mm})$ horizontally and $16 \mathrm{lp} / \mathrm{mm}$ vertically on the image sensor. The fact that resolving power in the vertical, or beam sweeping, direction is lower than the horizontal direction is not the result of drag as described in Sec. II B, rather the resolving power is determined by the width and height of the rectangular aperture. The measured resolution values correspond well to 


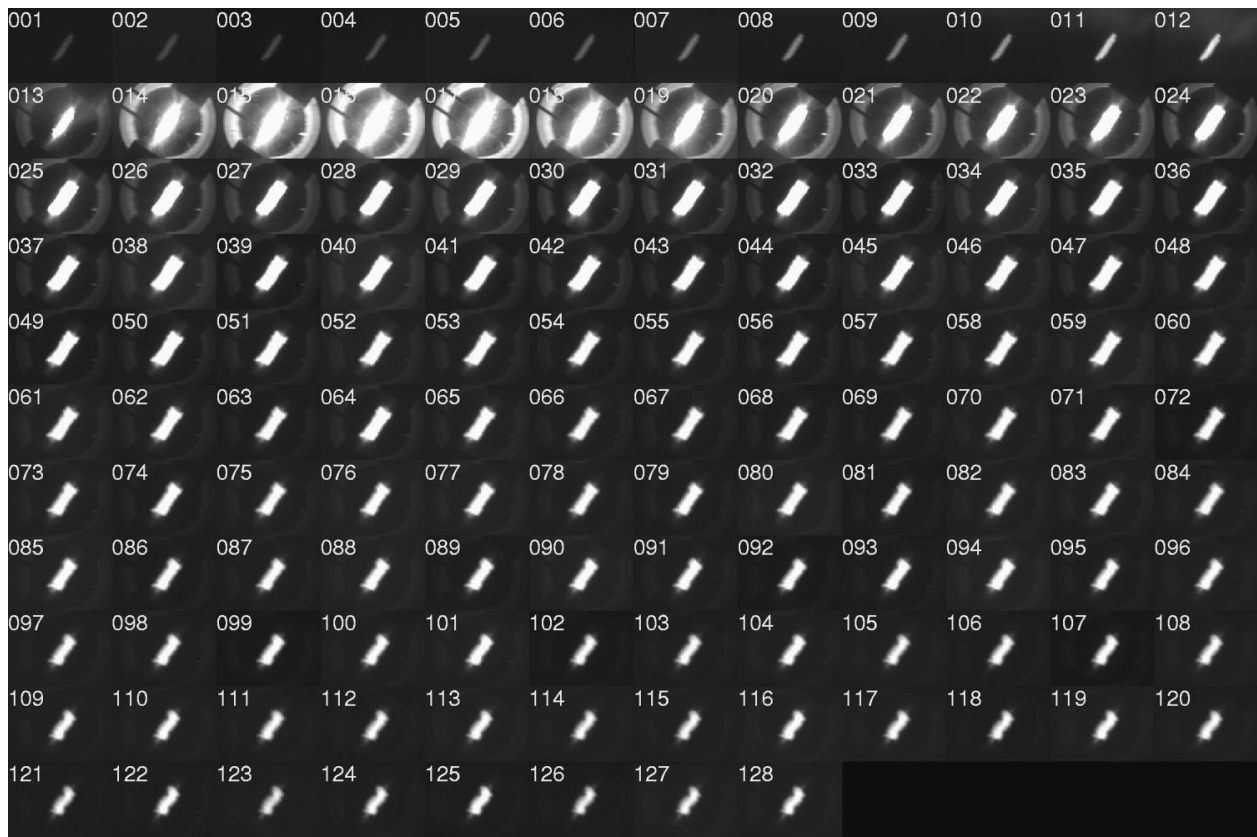

FIG. 15. A 6.2 Mfps recording of a single event in the xenon flash tube. The frame number is indicated on the top left corner of each frame. A preionization plasma channel can be seen in the first 12 frames and than the main discharge generates a bright flash lasting about $1.6 \mu \mathrm{s}$. The remaining plasma then dissipates in a swirling spiral pattern.

the specified resolutions of the framing camera's optics. Using an Olympus microscope with a $60 \times$ high resolution water immersion objective and a $2 \times$ magnifier, objects as small as $0.4 \mu \mathrm{m}$ wide can be clearly defined, see Fig. 13 .

Several micron-sized bubbles are imaged using the same microscope with both the Brandaris 128 and Imacon 468 cameras. The best frames obtained by each camera are reproduced in Fig. 14. While the image qualities are roughly comparable, the Imacon produced a somewhat noisy image, which is a result of the image intensifier.

\section{E. Examples}

An EG\&G FX-1163 (Perkin-Elmer Optoelectronics, Salem, MA) short arc xenon flash lamp was mounted directly on the primary image plane. An arc discharge event was recorded at 6.2 Mfps. The result is shown in Fig. 15. A pre- ionization plasma channel can be seen in the first 12 frames and then the main discharge generates a bright flash lasting about $1.6 \mu \mathrm{s}$. The remaining plasma then dissipates in a swirling spiral pattern.

The behavior of micron-sized bubbles in an ultrasound field was investigated using the setup described by de Jong et $a l .{ }^{13}$ A modified MVS-7010 (Perkin-Elmer) xenon discharge strobe was used as the illumination source. A burst of nine cycles ultrasound with a center frequency of $1.0 \mathrm{MHz}$ was used. Figure 16 shows a recording at $14.5 \mathrm{Mfps}$. The first cycle of ultrasound caused three small bubbles to be transformed into a cloud of larger bubbles, which then oscillate during the rest of the ultrasound excitation. In this event, radial oscillation, translation, fracturing of the bubble, and nonspherical oscillation were all observed in one continuous recording. The transient motions during the beginning and

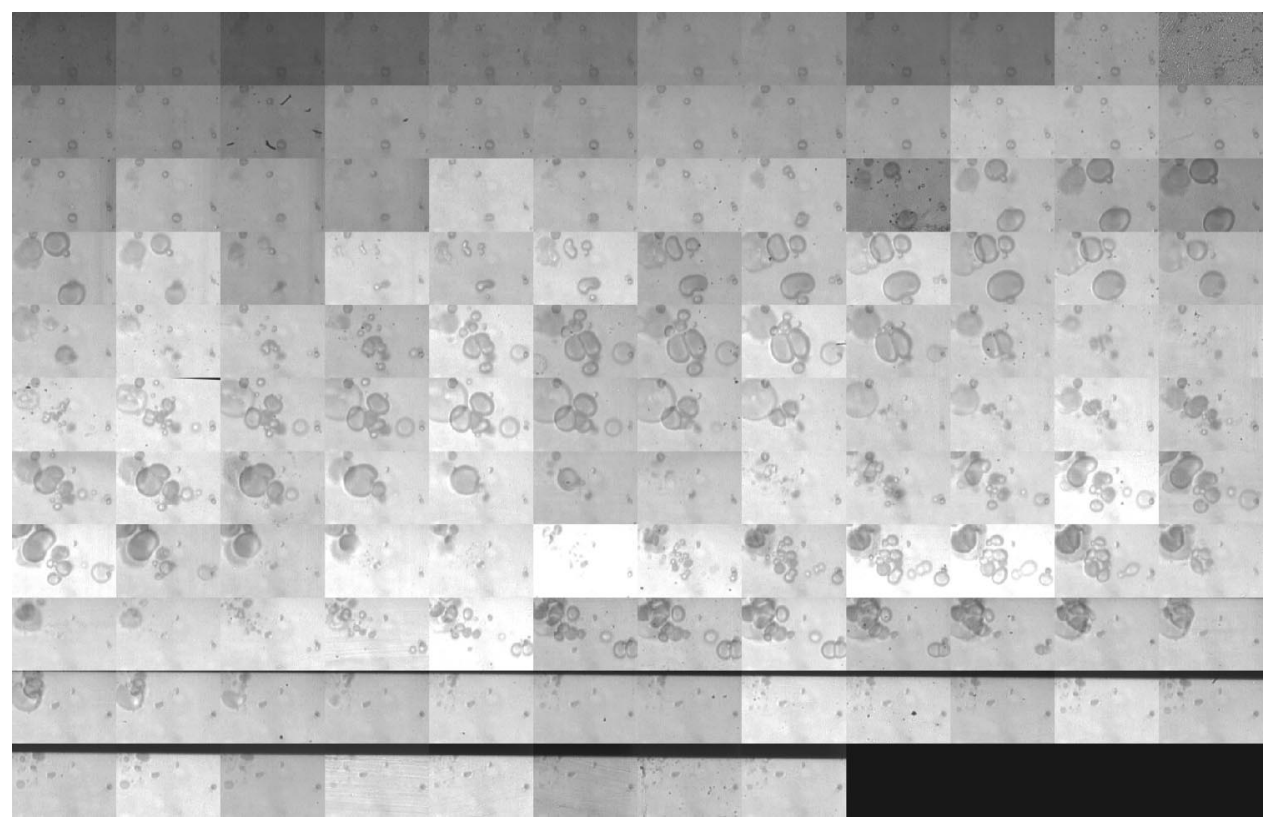

FIG. 16. A sequence of digital frames showing a portion of a full sequence. Shown are 128 frames of $227 \times 160$ pixels out of $500 \times 292$ pixels. The interframe time was $70 \mathrm{~ns}$ and the frames are arranged in the same order as in Fig. 15. Three small bubbles can be observed to transform into a cloud of larger bubbles and undergo several cycles of oscillation. The transient behavior during the beginning and the end of the ultrasound excitation can be clearly seen. 
TABLE I. Comparison of the main characteristics of Brandaris 128 to two commercially available camera types.

\begin{tabular}{|c|c|c|c|}
\hline & $\begin{array}{l}\text { Rotating } \\
\text { mirror }\end{array}$ & Electronic & $\begin{array}{c}\text { Brandaris } \\
128\end{array}$ \\
\hline & Photographic & Image intensifier & \\
\hline Media & Film & $+\mathrm{CCD}$ & $\mathrm{CCD}$ \\
\hline Framing rate (Mfps) & 25 & 200 & 25 \\
\hline No. of frames & 130 & 8 & 128 \\
\hline No. of runs & 1 & 2 & 6 \\
\hline Turnover time (seconds) & 1800 & 60 & 1 \\
\hline Total number of frames & 130 & 16 & 768 \\
\hline Time between runs & $60 \mathrm{~s}$ & $4 \mu \mathrm{s}$ & $20 \mathrm{~ms}$ \\
\hline Time between subsegments & N/A & N/A & $17 \mu \mathrm{s}$ \\
\hline
\end{tabular}

ending of the ultrasound excitation are also revealed, demonstrating the value of a long recording time. Other recorded examples are available as media files on the Brandaris web site. $^{28}$

\section{DISCUSSION}

The main characteristics of the Brandaris 128 high-speed framing camera are compared with commercially available high-speed cameras in Table I. While there exist technologies that outperform our system in frame rate or other specifications, Brandaris 128 offers a unique combination of the high frame rate and extended recording time of rotating mirror systems and the sensitivity and convenience of electronic cameras. Since image intensifiers were not used, the acquired frames have good image quality. Thanks to high sensitivity CCDs, the optical sensitivity was found to be only $50 \%$ lower than a camera employing image intensifiers. This camera is especially suited for studies of nonrepetitive or nonrepeatable events in the microsecond to nanosecond time scale.

\section{ACKNOWLEDGMENTS}

This study was financially supported by Fundamenteel Onderzoek der Materie (FOM) of The Netherlands and the Interuniversity Cardiology Institute of The Netherlands (ICIN). The authors would like to thank Sid Nebeker from Cordin Scientific Imaging for his scientific interest in this project. Technical contributions by the following individuals are also gratefully acknowledged: Cees Pakvis, Leo Bekkering, Wim van Alphen, Frans van Egmond, Jan Hankoop, Elmer Koene, and Fons Laan of Erasmus University; GertWim Bruggert, Rogier Nap, Edip Can, Ivo Heitman, and
Roger Jeurissen of the University of Twente; and finally, Jeroen Louwers and Martijn van Balen of AED Electronics (Eindhoven, The Netherlands).

${ }^{1}$ G. Debrégeas, P.-G. de Gennes, and F. Brochard-Wyart, Science 279, 1704 (1998).

${ }^{2}$ D. Bonn, H. Kellay, M. Prochnow, K. Ben-Djemiaa, and J. Meunier, Science 280, 265 (1998)

${ }^{3}$ R. da Silveira, S. Chaieb, and L. Mahadevan, Science 287, 1468 (2000).

${ }^{4}$ D. M. Mueth, G. F. Debregeas, G. S. Karczmar, P. J. Eng, S. R. Nagel, and H. M. Jaeger, Nature (London) 406, 385 (2000).

${ }^{5}$ M. Versluis, B. Schmitz, A. von der Heydt, and D. Lohse, Science 289, 2114 (2000)

${ }^{6}$ S. M. Deban and W. M. Olson, Nature (London) 420, 41 (2002).

${ }^{7}$ S. N. Fry, R. Sayaman, and M. H. Dickinson, Science 300, 495 (2003).

${ }^{8}$ B. Gabriel and J. Teissie, Biophys. J. 76, 2158 (1999).

${ }^{9}$ D. May, J. Allen, J. Gut, and K. Ferrara, in IEEE Ultrasonics Symposium, Atlanta (IEEE, New York, 2001), pp. 1385-1388.

${ }^{10}$ P. Marmottant and S. Hilgenfeldt, Nature (London) 423, 153 (2003).

${ }^{11}$ T. Kodama and K. Takayama, Ultrasound Med. Biol. 24, 723 (1998).

${ }^{12}$ W. Sass, M. Braunlich, H. Dreyer, E. Matura, W. Folberth, H. Priesmeyer, and J. Seifert, Ultrasound Med. Biol. 17, 239 (1991).

${ }^{13}$ N. de Jong, P. J. Frinking, A. Bouakaz, M. Goorden, T. Schourmans, X. Jingping, and F. Mastik, Ultrasound Med. Biol. 26, 487 (2000).

${ }^{14}$ J. Chomas, P. Dayton, D. May, J. Allen, A. Klibanov, and K. Ferrara, Appl. Phys. Lett. 77, 1056 (2000).

${ }^{15}$ M. Postema, A. Bouakaz, C. T. Chin, and N. de Jong, IEEE Trans. Ultrason. Ferroelectr. Freq. Control 50, 523 (2003).

${ }^{16}$ N. Kudo, T. Miyaoka, K. Kuribayashi, and K. Yamamoto, J. Acoust. Soc. Am. 108, 2547 (2000).

${ }^{17}$ T. G. Etoh, D. Poggemenn, A. Ruckelshausen, A. Theuwissen, G. Kreider, H.-O. Folkerts, H. Mutoh, Y. Kondo, H. Maruno, K. Takubo et al., in IEEE International Solid-State Circuits Conference, San Francisco (IEEE, New York, 2002), pp. 46-47.

${ }^{18}$ E. Muybridge and A. V. Mozley, Human and Animal Locomotion (Dover, New York, 1887).

${ }^{19}$ B. Brixner, in 20th International Congress on High Speed Photography and Photonics, edited by J. M. Dewey and R. G. Racca (SPIE, Bellingham, WA, 1992), Vol. 1801, pp. 52-60.

${ }^{20}$ G. W. Smith, M. J. Riches, R. B. Huxford, P. A. Smith, C. L. Seymour, and J. D. Bell, in 24th International Congress on High-Speed Photography and Photonics, edited by K. Takayama, T. Saito, H. Kleine, and E. V. Timofeev (SPIE, Bellingham, WA, 2001), Vol. 4183, pp. 105-118.

${ }^{21}$ M. J. Riches and B. R. Garfield, in Ref. 19, pp. 12-19.

${ }^{22}$ Named after the famous Brandaris lighthouse at Terschelling, The Netherlands, see http://www.vuurtorens.net/.

${ }^{23}$ V. Parker and C. Roberts, in High Speed Photography and Photonics, edited by S. F. Ray (SPIE, Bellingham, WA, 2002), Vol. PM120, pp. $158-180$.

${ }^{24}$ E. A. Igel and M. Kristiansen, Rotating-Mirror Streak and Framing Cameras (SPIE, Bellingham, WA, 1997), Vol. PM43.

${ }^{25}$ A. K. Jain, Fundamentals of Digital Image Processing, 1st ed. (PrenticeHall, Englewood Cliffs, NJ, 1988).

${ }^{26} \mathrm{R}$. McLenaghan and S. Levy, in CRC Standard Mathematical Tables and Formulae, edited by D. Zwillinger (CRC, Boca Raton, FL, 1995), pp. 265-266.

${ }^{27}$ Tech. Rep. ISO 5800, International Organization for Standardization, 1987.

${ }^{28}$ The Brandaris 128 website: http://www.brandaris128.nl/. 